\title{
ARRHYTHMIAS
}

\section{Refined programming accentuates the benefits of ICDs}

The appropriate functioning of implantable-cardioverter defibrillators (ICDs) can substantially reduce mortality, but the inappropriate discharge of these devices, for example triggered by supraventricular tachycardias, is frequent and confers a substantial burden of morbidity on patients. Data from MADIT-RIT, presented at the AHA 2012 Scientific Sessions and published in the New England Journal of Medicine, indicate that, by adjusting the ICD algorithm to differentiate between ventricular tachyarrhythmias and other, nonlifethreatening arrhythmias, morbidity and mortality can be substantially reduced.

Conventional ICDs are programmed to discharge an electric shock after a 2.5-s delay at a heart rate of 170-199 bpm, and after a 1.0 -s delay at $\geq 200 \mathrm{bpm}$. The MADIT-RIT investigators randomly allocated 1,500 patients to receive an ICD with one of three programmes-conventional, highrate (2.5-s delay at $\geq 200 \mathrm{bpm}$ ), or delayed (60.0-s delay at 170-199bpm, 12.0-s delay at 200-249 bpm, and 2.5-s delay at $\geq 250 \mathrm{bpm}$ ).
During follow up (mean 1.4 years), the primary end point (first occurrence of inappropriate antitachycardia pacing or shocks) occurred in 20\%, $4 \%$, and 5\% of patients in each group, respectively (compared with conventional therapy, HR $0.21,95 \%$ CI $0.13-0.34, P<0.001$ with high-rate therapy; HR $0.24,95 \% \mathrm{CI}$ $0.15-0.40, P<0.001$ with delayed therapy). Mortality was significantly decreased with high-rate therapy (HR 0.45, 95\% CI $0.24-0.85, P=0.01$ ) and nonsignificantly decreased with delayed therapy (HR 0.56, 95\% CI 0.30-1.02, $P=0.06$ ). The trial investigators note that "the mechanisms linking diminished occurrences of inappropriate shock ... with reduced mortality are complex and require further investigation to determine specific causality."

In an Editorial that accompanied the trial report, Dr Bruce Wilkoff concludes that "the value of carefully choosing tachycardiadetection parameters is demonstrated definitively. The overriding principle is to be certain that there is a sustained tachyarrhythmia before treating the rhythm.

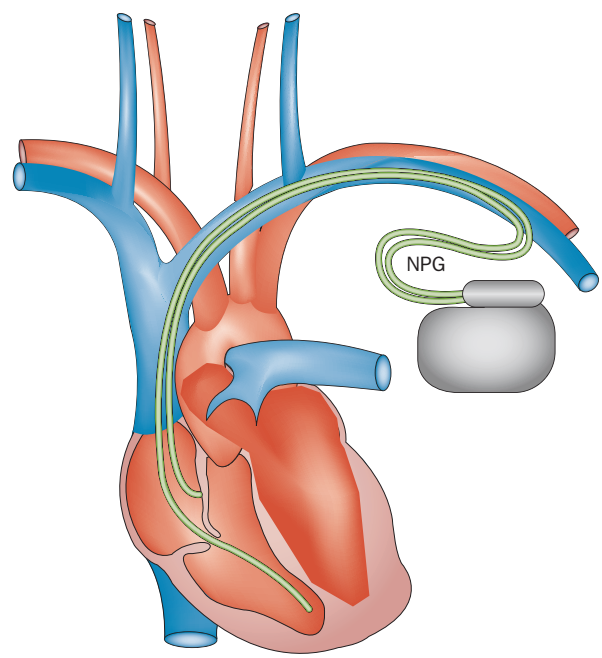

This principle will ... systematically reduce overall morbidity and improve survival."

Gregory B. Lim
Original article Moss, A. J. et al. Reduction in inappropriate therapy and mortality through ICD programming. N. Engl. J. Med. doi:10.1056/NEJMoa1211107 